\title{
Immunoresponse to Allogeneic Synovial or Xenogenic Mesenchymal Stromal Cells in a Co-Culture Model
}

\author{
Seth S. Jump ${ }^{1,2}$, David S. Smith ${ }^{1}$, David C. Flanigan ${ }^{3}$, Alicia L. Bertone ${ }^{1,2}$ \\ ${ }^{1}$ Comparative Orthopaedic Research Laboratories, The Ohio State University, Columbus, USA \\ ${ }^{2}$ Department of Veterinary Biosciences, College of Veterinary Medicine, The Ohio State University, Columbus, USA \\ ${ }^{3}$ Department of Orthopaedics, College of Medicine, The Ohio State University, Columbus, USA \\ Email: bertone.1@osu.edu
}

Received February 2, 2012; revised March 7, 2012; accepted March 16, 2012

\begin{abstract}
The purpose of our investigations was to measure, in a co-culture condition, the immunoresponse to allogeneic or xenogenic cells, selected as potential sources for cell therapy of arthritis. We challenged human spleen-derived cells (hSpl) by three different mechanisms: 1) exposure to donor allogeneic or xenogeneic cellular antigens; 2) exposure to donor cells transduced with adenoviral antigens (Ad) and 3) lipopolysaccharide (LPS), a known inflammatory immunostimulant. The immunoresponse to allogeneic human synovial-derived mesenchymal stromal cells alone or transduced with adenoviral green fluorescent protein (hSD-MSC or hSD-MSC/GFP) or the immunoresponse to xenogeneic equine mesenchymal stromal cells (eqMSC) or equine dermal fibroblasts (eqDFb), characterized by the proportion of $\mathrm{CD}^{+}$, $\mathrm{CD}^{+}$, and $\mathrm{CD}^{+}$human splenocytes (hSpl), was measured on Day 0 and Day 6 of co-culture by flow cytometry. In culture with hSD-MSC, hSD-MSC/GFP, eqDFb, or eqMSC, the proportion of $\mathrm{CD3}^{+}$and $\mathrm{CD}^{+} \mathrm{hSpl}$ increased with time in culture but not with exposure to cell allo- or xeno-antigens. Both hSD-MSC and hSD-MSC/GFP increased in number during culture and were not affected in viability or proliferation by co-culture with allogeneic hSpl. In this in vitro, primary exposure study, hSpl demonstrated a natural selection and adaptation to a short-term cell culture environment, and that neither allogeneic nor xenogeneic cell antigens incited a greater cellular immunoactivation than co-cultured hSpl alone.
\end{abstract}

Keywords: Arthritis; Stem Cells; Allogeneic; Xenogeneic; Immunoresponse

\section{Introduction}

Cutting-edge advancements in regenerative medicine may harness the potential of an engineered cell source as a therapeutic vector for the repair and restoration of multiple human tissues including articular cartilage damaged by injury or degenerated through either osteoarthritis or rheumatoid arthritis. Allogeneic cells, from a different organism of the same species or xenogenic cells, from a different species, could provide a nearly limitless supply of therapeutic cells for use in tissue repair. Allogeneic or xenogeneic cell sources, engineered to serve as a clinical tool, could dramatically and irrevocably enhance current medical practices that promote healing.

Allogeneic mesenchymal stem cells in Phase III clinical trials, have been used as a treatment for inflammatory conditions including acute Bone Versus Graft Disease and Crohn's disease [1]. In orthopaedic medicine, autologus cell-based strategies for tissue repair and restoration, including autologous chondroctye implantation and osteochondral grafts, are currently used in clinical practice with some promising results [2-6]. A potentially promis- ing alternative or adjunct strategy to current regenerative techniques could use allogeneic cells or xenogenic cells. To this end, a cell-based allogeneic therapy has been provided for 1st generation, commercially-available cartilage neograft (DeNovo ${ }^{\circledR}$ ET Live Chondral Engineered Tissue Graft, Zimmer Holdings, Inc). This product, approved last year, relies on allogeneic, juvenile chondrocytes and a proprietary cell-scaffold system to promote healing. Potentially, allogeneic or xenogeneic cells could serve as effective therapeutic vectors in vivo to integrate into the native biological environment of tissue. The immunoresponse of allogeneic and xenogeneic cells needs to be further investigated, and further observations of the cellular mechanisms and interactions will help to elucidate and describe the immunoresponse for future clinical trials.

New cell sources could improve the repair and restoration of articular cartilage, such as in injured cartilage or deteriorating cartilage as in osteoarthritis (OA), the most common form of arthritis. It is believed that there are immune processes that could be responsible for the de- 
generation of cartilage. In particular, activated immune cell infiltration, including T-cells, has been associated with the advancement of arthritis [7-9]. Finding a potential cell- or tissue-based treatment for damaged cartilage is dependent upon further understanding the immunoresponse of cells from an articular joint, including synovial lining cells or chondrocytes. Organ or cell transplantation is characterized by an activation of host defenses including the activation and proliferation of immune cell types including cluster determination $3\left(\mathrm{CD}^{+}\right)$mature lymphocytes, cluster determination $4\left(\mathrm{CD}^{+}\right)$T-helper (TH1) cells, and cluster determination $\left(\mathrm{CD}^{+}\right)$natural killer/ cytotoxic T lymphocytes (CTL) [7-9].

Allogeneic synovial-derived cells (SDSCs) have been successful in the repair of full-thickness defects of the femoral condyle in rats; however, contaminating macrophages provided evidence of a delayed immune reaction to the transplantated allogeneic cells [10]. Furthermore, xenogeneic SDSCs failed to repair cartilage defects in vivo, and an enhanced immune response, characterized by detection of major histocompatability complex antigen II (MHCII) in foreign bodies found in the repair tissue [11]. In cell culture, a limited number of studies have used a mixed immune cell design to evaluate host versus donor reactions [12-16]. Allogeneic human MSCs derived from bone marrow successfully reduced $\mathrm{CD}^{+}$expansion in cell culture providing support for the beneficial immunomodulation of MSCs [17], and allogeneic fibroblast-like synoviocytes have also reduced proliferation in T-cells found in bone marrow [12]. On the other hand, allogeneic peripheral blood mononuclear cells elicited an activation of both $\mathrm{CD}^{+}$and $\mathrm{CD}^{+}$cells [14]. Further characterization of the potential cellular activation of allogeneic or xenogeneic cells with the host immune system is needed to further develop clinical tools to control or monitor this reaction for the future of cell therapy as a treatment for joint disease. Our study will extend the findings in the literature by using another source of human immune cells, splenic tissue, to investigate the potential immune activation of $\mathrm{CD}^{+}, \mathrm{CD}^{+}$, and $\mathrm{CD}^{+}$cell types to allogeneic or xenogeneic mesenchymal stromal cells.

The purpose of our investigations was to measure, in a co-culture condition, the immunoresponse to allogeneic or xenogenic cells, selected as potential sources for cell therapy of arthritis. We challenged human spleen-derived cells (hSpl) by three different mechanisms: 1) exposure to donor allogeneic or xenogeneic cellular antigens; 2) exposure to donor cells transduced with adenoviral antigens (Ad); and 3) lipopolysaccharide (LPS), a known inflammatory immunostimulant. For the allogeneic experiment, human synovial-derived mesenchymal stromal cells (hSC-MSC) or hSC-MSC transduced with adenovirus expressing green fluorescent protein (hSC-MSC-
GFP) were co-cultured with allogeneic hSpl. In the xenogeneic experiment, equine (eq) bone-marrow derived MSCs (eqMSC) or equine dermal fibroblasts (eqDFb) were co-cultured with hSpl. Our hypothesis was that a cell-mediated challenge of either allogeneic or xenogeneic cells would stimulate the formation and development of $\mathrm{CD}^{+}$and $\mathrm{CD}^{+} \mathrm{hSpl}$ in co-culture compared to unchallenged hSpl and that adenoviral challenge may further enhance this effect. Additionally, the viability of the allogeneic or xenogeneic donor cells was expected to be reduced when co-cultured with human host cells.

\section{Methods}

\subsection{Human and Equine Donor Tissue Harvest and Digestion}

Synovial biopsies were obtained from the knee joint of orthopedic patients undergoing anterior cruciate ligament reconstruction by an author surgeon (DCF). Tissue harvest was conducted in accordance with the guidelines set by the Institutional Review Board (IRB Protocol 2009H0256) at The Ohio State University and only by consent of the patient.

Synovial biopsies were digested in sterile-filtered $(0.2$ um) media containing collagenase type I $(0.2 \% \mathrm{~m} / \mathrm{v})$ for 90 min (Gibco, Carlsbad, CA). Following digestion, cells were filtered through a cell strainer $(70 \mu \mathrm{M})$ and washed twice in Gey's Balanced Salt (GBSS) (Sigma-Aldrich, St. Louis). Before initial seeding, cell samples from synovium were exposed to trypan blue exclusion stain and cell count and cell viability was determined using a hemacytometer

Bone marrow aspirates were obtained from the sternum of adult horses immediately after euthanasia for reasons unrelated to the immune system. A bone marrow aspiration needle (MD Tech Inc, Gainesville, FL) was inserted into a sternebral body from the ventral aspect of the sternum, and marrow was aspirated into a sterile, heparin-flushed (American Pharmaceutical Partners Inc., Schaumburg, IL), 12-mL syringe. The procedure was repeated until a minimum of $10 \mathrm{~mL}$ of bone marrow was collected. Primary BMD-MSCs were isolated via centrifugation of marrow specimens and cultured in a monolayer, as has been described [18]. Derived eqBMD-MSCs (eqMSC) were confirmed as pluripotent by culturing in controlled osteogenic, chondrogenic, and adipogenic media containing dexamethasone with ascorbate, recombinant human transforming growth factor-I, and dex methasone with insulin and indomethacin (Gibco, Grand Island, NY) [19-21].

Dermal fibroblasts (DFb) were obtained via skin biopsy as part of another equine study [20,22]. Full-thickness skin tissue was harvested using a $5 \mathrm{~mm}$ diameter biopsy punch from the pectoral region (10 - 12 punches 
per horse) from each of six adult horses. The dermal layer was dissected from the epidermis under a microscope, and DFbs were isolated by type- 1 collagenase digestion (GIBCO, Grand Island, NY) and cultured in DMEM supplemented with L-glutamine (300 mg/mL), penicillin (30 mg/mL), streptomycin (30 mg/mL), and $10 \%$ fetal bovine serum at $37^{\circ} \mathrm{C}$ in a $5 \% \mathrm{CO}_{2}$ atmosphere.

Synovial-derived mesenchymal stromal cells were passaged a minimum of 4 times, but no more than 7 times. Previous studies in the literature have demonstrated that hSD-MSCs cells maintain a consistent phenotype between passages 3 and 8 [23,24]. Equine BMD- MSC and Dfb were low passage ( $<3$ passages).

\subsection{Tissue Harvest of Host Mixed Immune Cells}

Human spleens were selected as the host mixed cell population for co-culture experiments. Approval for receiving portions of human spleens was granted by Lifeline of Ohio, an organ donor center in Columbus, Ohio. Splenocytes were harvested and prepared with high yield and successfully grown in cell culture using methods adapted from murine splenocyte culture [25,26]. Briefly, tissue was trimmed into small pieces and thoroughly minced using a syringe plunger inside of a nylon cell strainer $(70 \mu \mathrm{m})$ in a $35 \mathrm{~mm}$ cell culture plate. Cells were digested using Ack lysing buffer (Gibco, Carlsbad, CA), and the samples were subjected to consecutive washes in ice cold PBS. Isolated splenocytes were immediately allocated to cultures as described below in experimental design.

\subsection{Adenoviral Transduction of Donor Allogeneic Synovial-Derived Mesenchymal Stromal Cells}

Recombinant, E1-deficient, serotype-5 adenovirus vectors containing the open reading frame segment of human GFP (AdGFP) under the control of the cytomegalovirus promoter were generated. Successful transduction of AdGFP was verified in cell culture. Viral titer [infection units per $\mathrm{mL}$, (IFU/mL)] was determined (Adeno-X Rapid TiterKit; Clonetech, Mountain View, CA, USA) and SD-MSCs were transduced at 100 multiplicities of infection (MOI) or $1 \times 102$ infectious units per cell [1921].

\subsection{Cell Co-Culture Conditions and Experimental Design}

For the overall experimental design, three sources of donor cells, hSD-MSC (allogeneic; Experiment 1) and eqMSC and eqDFb (xenogeneic; Experiment 2), were seeded at 10,000 cells/cm2 onto 24-well cell culture plates at Day -2 in $10 \%$ FBS containing $1 \%$ penicillin streptomycin in
DMEM (Invitrogen). Human SD-MSC cultures were transduced with Ad-GFP on Day -1. For Experiments 1 and 2, isolated hSpl were obtained from 3 different human spleens for each experiment (6 spleens) and cultured in triplicate alone or added to donor cultures at a 50:1 ratio or $500,000 \mathrm{hSpl} / \mathrm{cm}^{2}$ on Day 0. For Experiment 1, hSpl were added to one of three co-culture immune challenges at Day 0; allogeneic hSD-MSC, allogeneic hSDMSC-GFP, or lipopolysaccharide stimulation (LPS 10 $\mu \mathrm{g} / \mathrm{mL}$, Sigma-Aldrich, St. Louis, MO). For Experiment 2, hSpl were assigned to one of three co-culture immune challenges at Day 0; xenogeneic eqMSC, xenogeneic eqDFb, or lipopolysaccharide stimulation (LPS $10 \mu \mathrm{g} / \mathrm{mL}$, Sigma-Aldrich, St. Louis, MO). On Day -1, hSD-MSC wells were assigned to receive adenoviral vector transduction. Cells were transduced with AdGFP at $100 \mathrm{MOI}$ for 2 h in Gey's Balanced Salt Solution. Effective transduction methods with AdGFP have been validated in previously published work [19]. On Day 0, prior to the addition of the allogeneic hSpl cells, GFP expression was confirmed by fluorescent microscopy and recorded. In addition to co-cultures, hSpl were cultured alone. At day 0 , hSpl were removed for cellular viability staining and flow cytometry analysis approximately two hours after co-cultures were established to serve as a baseline immune status. At Days 0 and 6, hSpl were harvested for cell surface marker content as measured by flow cytometry.

\subsection{Flow Cytometry, Cell Numbers, and Cell Viability}

Flow cytometry (C-Flow Plus, Accuri, Ann Arbor, MI) was performed on hSpl subjected to extracellular staining to determine the proportion of cells positively-stained for binding of CD3, CD4, or CD8 antibodies. Human monoclonal antibodies for each immune cell subtype (FITCconjugated-anti-CD3, APC-conjugated anti-CD4, and PEconjugated anti-CD8, R\&D Systems, Minneapolis, MN) were used to assess the potential formation of immune cell types. At Days 0 and 6, we investigated formation of $\mathrm{CD}^{+}, \mathrm{CD}^{+}$, and $\mathrm{CD}^{+}$cells; an unstained control and an antibody control for each respective antibody were used on each of flow cytometry analysis.

Cell number and viability for both hSD-MSC and hSD-MSC/GFP were determined on Day 6. Cells were trypsinized, washed, and subjected to a trypan blue exclusion stain. Cells were counted in a hemacyometer; the number of live cells was recorded.

\subsection{Statistical Analysis}

For co-culture experiments, cells isolated from one human joint (hSD-MSC or hSD-MSC/GFP or one animal (eqMSC or eqDFb) and an individual spleen (hSpl) were 
considered an n of 1. Data for quantitative outcomes of $\mathrm{hSpl} \mathrm{CD} 3^{+}, \mathrm{CD}^{+}, \mathrm{CD}^{+}$number and proportion, as well as donor cell number and viability were presented as means \pm standard error of the mean (SEM). Two factor analysis of variance (ANOVA) for Day (0 and 6) and hSpl condition (alone, hSD-MSC, hSD-MSC-GFP; or alone, eqMSC, eqDFb, LPS) was performed for hSpl number, donor cell number, and hSpl or donor cell viability. All analyses were performed using a comercially available statistical software package (Statistical Analysis Software, SAS 9.1). Statistical significance was accepted at $\mathrm{p}<0.05$

\section{Results}

Human spleens were obtained on ice, and splenoctyes were harvested with initial high hSpl number and viability (>95\%) (Figure 1(A)) and co-cultured with donor cells at Day 0 (Figure 1(B)). The proportion of gated viable hSpl on the Day 0 of culture measurement by flow cytometry was in the range of $65 \%-74 \%$ (Figures 2(a) and 2(b)). The proportion of $\mathrm{CD}^{+} \mathrm{hSpl}\left(\mathrm{CD}^{+}, \mathrm{CD}^{+}\right.$, plus $\mathrm{CD} 4^{+}$) was $<15 \%$ of total gated cells on Day 0 culture measurement by flow cytometry (Figures 3 and $\mathbf{4}$ ). Culture of hSpl alone resulted in a significant decrease in hSpl numbers and viability to an average of approximately $31 \%$ by Day 6 as measured by flow cytometry (Figures 2(a) and 2(b)). Co-culture of hSpl with allogeneic or xenogeneic donor cells did not further decrease hSpl numbers (Figures 2(a) and 2(b)). Both allogeneic and xenogeneic donor cells proliferated in cell co-culture with hSpl (Figure 5) and were of normal morphology with high efficiency of GFP expression demonstrated in hSD-MSC-GFP cells for the entire 6 days. (Figure 1(B), insert) Synovial-derived mesenchymal stromal cells were successfully transduced (hSD-MSC-GFP) 24h after transduction with AdGFP at 100 multiplicities of infection (100 MOI). (Day 0).

In the allogeneic co-culture Experiment 1, the proportion of $\mathrm{CD3}^{+}$and $\mathrm{CD}^{+} \mathrm{hSpl}$ was significantly greater on Day 6 (4-fold and 5-fold, respectively) regardless of culture condition as compared to Day 0. (Figures 3(a) and 3 (b)) On Day 6, hSpl cultured in LPS had a greater proportion of $\mathrm{CD}^{+} \mathrm{hSpl}$ than hSpl cultured alone. There was no change in the proportion of $\mathrm{CD}^{+} \mathrm{hSpl}$ on Day 6 versus 0 (Figure 3(c)).

In the xenogeneic co-culture Experiment 2, the proportion of $\mathrm{CD}^{+}$and $\mathrm{CD}^{+} \mathrm{hSpl}$ was significantly greater on Day 6 (3-fold and 6-fold, respectively) regardless of culture condition as compared to Day 6 as in the allogeneic co-culture experiment and compared to Day 0. (Figures 4(a) and 4(b)) On Day 6, hSpl co-cultured with eqDFb had a lesser proportion of $\mathrm{CD}^{+} \mathrm{hSpl}$ than hSpl cultured alone. There was no change in the proportion of $\mathrm{CD} 4^{+}$
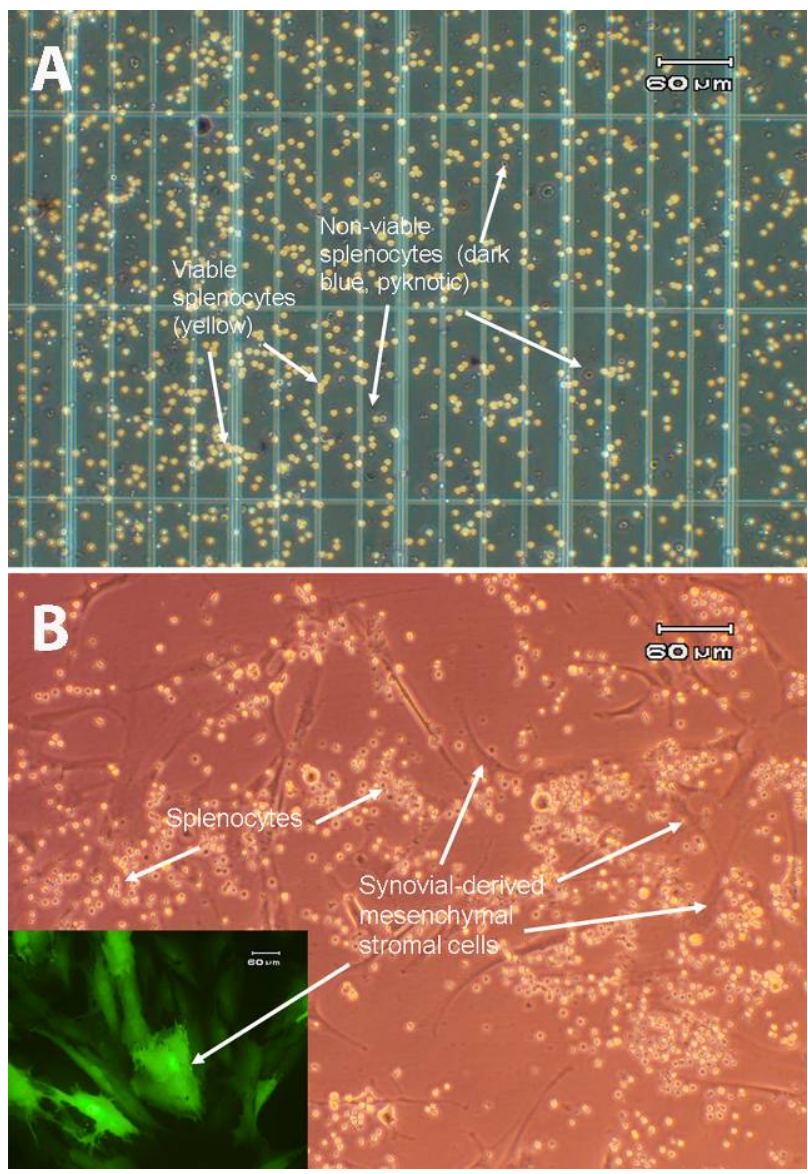

Figure 1. (A) Photomicrograph (100× magnification) of a hemacytometer containing a cell dilution of human splenocytes stained with trypan blue stain after being digested from a human spleen. Viable hSpl are yellow and circular, and non-viable human splenocytes $(\mathrm{hSpl})$ are blue and pyknotic. Each spleen sample that was digested had greater than $95 \%$ living cells prior to the start of the co-culture experiments (Day 0); (B) Photomicrograph (100× magnfication) of a co-culture containing hSpl (small circular cells) and human synovial-derived mesenchymal stromal cells (hSD-MSC) 100x magnification. Human SD-MSC transduced with AdGFP at 100 infectious Ad particles per cell, had $>95 \%$ expression of GFP under fluorescent microscopy at 24 hours (insert; $200 \times$ magnification).

cells between days (Figure 4(c)).

\section{Discussion}

This study provided evidence that, in a short-term culture environment, hSpl undergo a natural selection and adaptation to cell culture conditions. In the allogeneic or xenogenic co-culture conditions, no particular evaluated cell source (allogeneic synovial or xenogeneic bonemarrow, or skin) or donor source (human allogeneic or equine xenogeneic) promoted survival, inhibited death, or promoted death of the lymphocyte cell fraction of hSpl. In addition, these data showed that neither allogeneic nor 


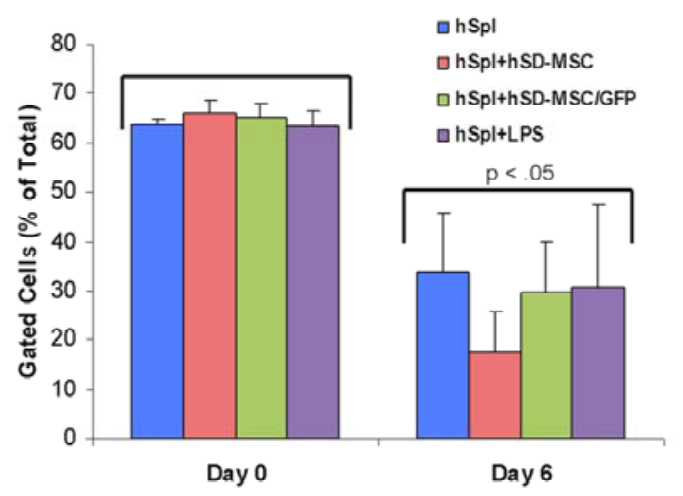

(a)

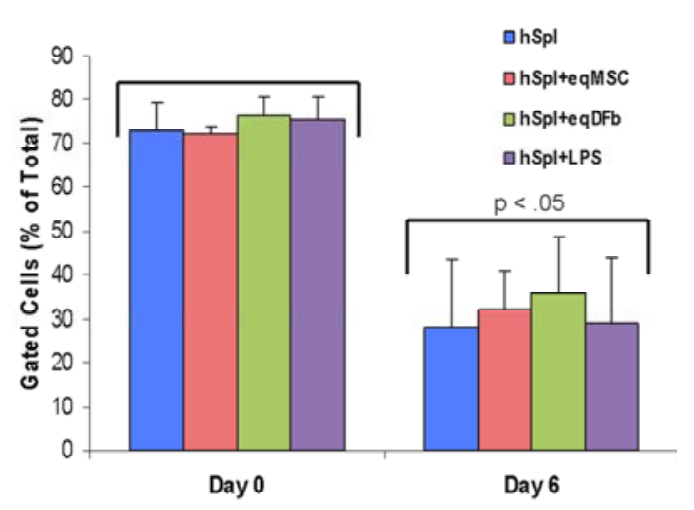

(b)

Figure 2. Number of gated (viable) hSpl on Day 0 and Day 6 in the allogeneic Experiment 1 (Panel (a)) and the xenogeneic Experiment 2 (Panel (b)). Abbreviations: hSpl = human splenocytes, hSpl + hSD - MSC $=$ human splenoctyes cocultured with human synovial-derived mesenchymal stromal cells, hSpl + hSD - MSC/GFP = human splenocytes cocultured with human synovial-derived mesenchymal stromal cells transduced with AdGFP, hSpl + eqMSC = human splenoctyes co-cultured with equine mesenchymal stromal cells, hSpl + eqDFb = human splenocytes co-cultured equine dermal fibroblasts, hSpl + LPS = human splenocytes cocultured with lipopolysaccharide (LPS, $10 \mu \mathrm{g} / \mathrm{mL}$ ). Data are mean \pm SEM. The bracket $(p<0.05)$ denotes a statistical difference at Day 6 compared to Day 0.

xenogeneic cells elicited a lymphocytic/dendritic cell selection and no donor source promoted proliferation, at least in vitro and for this short duration of primary exposure. The short survival of immune cells in culture (Spl or peripheral blood monocytes) limit in vitro studies. However, within these limitations, the evidence suggested that allogeneic or xenogeneic cells may be immunotolerated, at least initially, in a host environment. Our study was a first to investigate the immunoresponse of a mixed population of hSpl to different tissue sources of both allogeneic and xenogeneic origin cells and assessed three differing mechanisms of immune challenge, including 1) cellular antigens, 2) adenoviral antigens, and 3) lipopolysaccharide (LPS), a mediator of the inflammatory immune reaction. We were unable to document

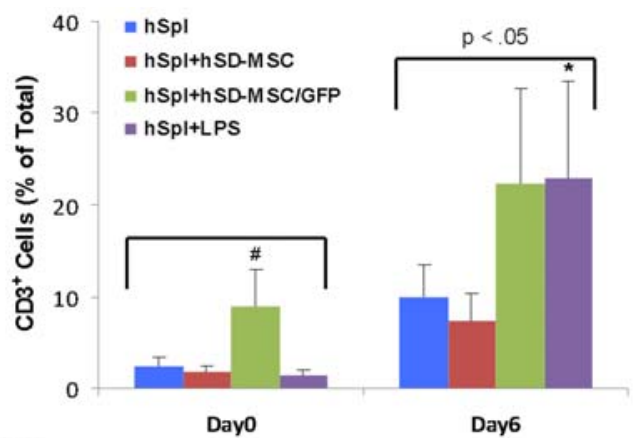

(a)

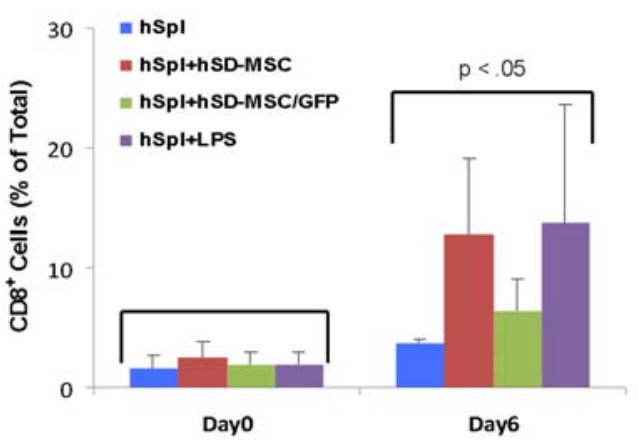

(b)

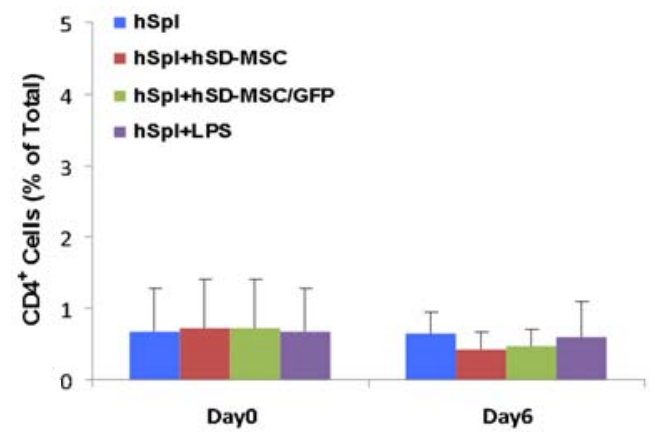

(c)

Figure 3. Human Human splenocytes (hSpl) co-cultured with allogeneic synovial-derived mesenchymal stromal cells (hSDMSC) and subjected to extracelluar staining using antibodies specific for CD3, CD4, or CD8. The proportion of positive cells for each antibody was determined by flow cytometry. (a) $\mathrm{CD3}^{+} \mathrm{hSpl}$ on Day 0 and Day 6. (b) $\mathrm{CD8}^{+} \mathrm{hSpl}$ on Day 0 and Day 6. (c) $\mathrm{CD4}^{+} \mathrm{hSpl}$ on Day 0 and Day 6. Abbreviations: hSpl = human splenocytes, hSpl + hSD MSC = human splenoctyes co-cultured with human synovial-derived mesenchymal stromal cells, hSpl + hSD - MSC/ GFP = human splenocytes co-cultured with human synovial-derived mesenchymal stromal cells transduced with AdGFP, hSpl + LPS = human splenocytes co-cultured with lipopolysaccharide (LPS, $10 \mu \mathrm{g} / \mathrm{mL}$ ). Data are mean \pm SEM. The bracket $(p<0.05)$ denotes a statistical difference at Day 6 compared to Day 0 . \# denotes an unexplained difference compared to other groups at Day 0. ${ }^{*}$ denotes that hSpl + LPS at Day 6 is significantly greater than Day 0.

significant activation of $\mathrm{CD}^{+}$immune cells or death of immune cells or death of allogeneic or xenogeneic donor 


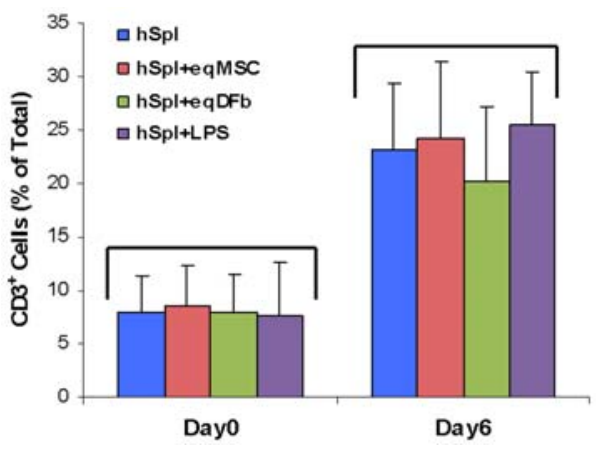

(a)

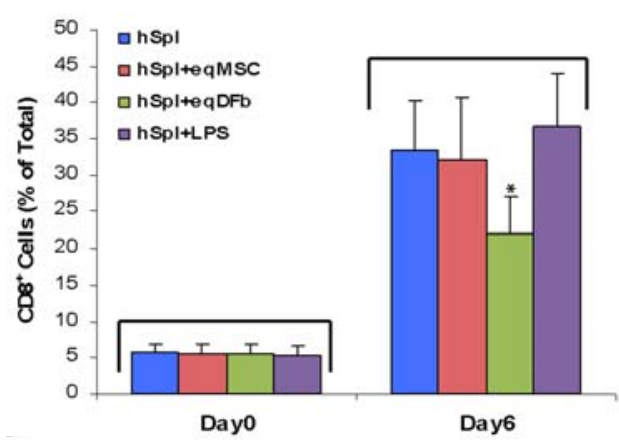

(b)

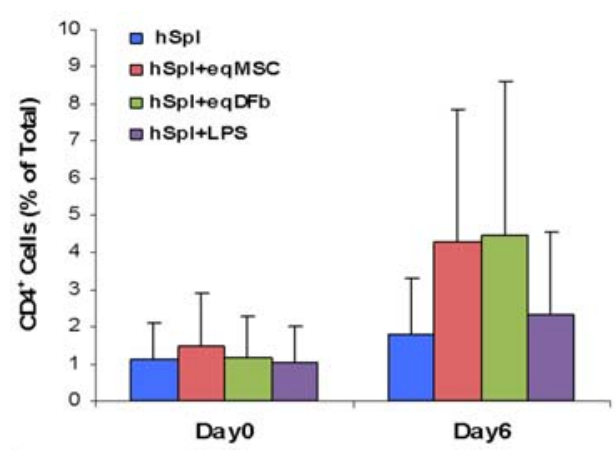

(c)

Figure 4. Human splenocytes (hSpl) co-cultured with xenogeneic equine bone-marrow derived mesenchymal stromal cells (eqMSC) or dermal fibroblasts (eqDFb) and subjected to extracelluar staining using antibodies specific for CD3, CD4, or CD8. The proportion of positive cells for each antibody was determined by flow cytometry. (a) $\mathrm{CD}^{+} \mathrm{hSpl}$ on Day 0 and Day 6. (b) $\mathrm{CD8}^{+} \mathrm{hSpl}$ on Day 0 and Day 6. (c) $\mathrm{CD4}^{+}$hSpl on Day 0 and Day 6. Abbreviations: hSpl $=$ human splenocytes, hSpl + eqMSC = human splenoctyes cocultured with equine mesenchymal stromal cells, hSpl + eqDFb = human splenocytes co-cultured equine dermal fibroblasts, hSpl + LPS = human splenocytes co-cultured with lipopolysaccharide (LPS, $10 \mu \mathrm{g} / \mathrm{mL}$ ). Data are mean \pm SEM. The bracket $(p<0.05)$ denotes a statistical difference at Day 6 compared to Day 0. *denotes that hSpl + EqDFb was significantly lower than other groups at Day 6.

cells. Dermal fibroblasts have not been stated to be immunoprivileged as has been claimed for the synovial fibroblast or bone-marrow derived mesenchymal pro-

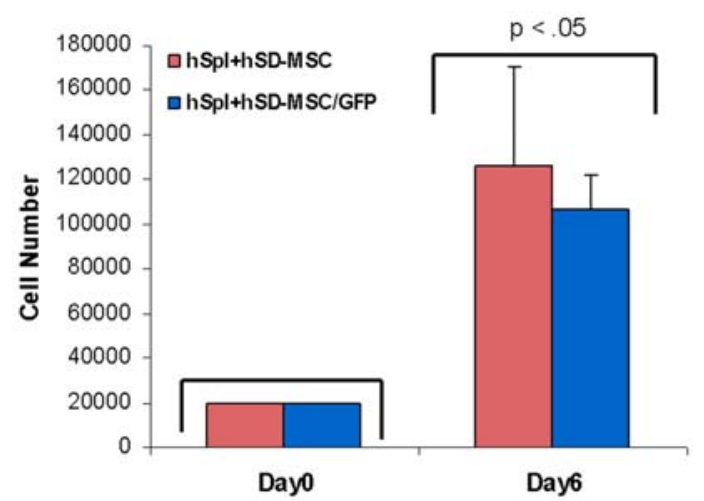

Figure 5. Cell number of human synovial-derived mesenchymal stromal cells (hSD-MSC) and human synovial-derived mesenchymal stromal cells transduced with adenovirus containing green fluorescent protein (hSD - MSC/GFP) on Day 0 and Day 6 as determined by hemacytometer live cell count following a trypan blue exclusion staining Data are mean \pm SEM. The bracket $(p<0.05)$ denotes a statistical difference at Day 6 compared to Day 0.

genitor and stromal cells, but our data suggested that xenogeneic dermal fibroblasts may be immunosuppressive to $\mathrm{CD}^{+}$cells and would warrant further investigation.

Importantly, further experiments in vivo would be necessary to more critically assess immunotolerance, to include longer exposure to antigens, and evaluate a second exposure to elucidate the cellular contributions and anamnestic response to isolated allogeneic or xenogeneic cells. To our knowledge, our splenocyte co-culture system is novel and contributed initial information on tolerance of these mixed immune cells to donor cells on short term exposure and supports previous publications claiming allogeneic juvenile chondroctyes exhibit immunotolerance [27]. Previous work has also provided evidence that synovial cells have immune privilege and will not be rejected by a host organism [28,29]. Some studies supported the tolerance of allo- or xenotransplants for tissue healing [30,31]. An additional study showed that xenogeneic chondrocytes can successfully repair full-thickness cartilage defects [32]. However, in a recent 2010 study, xenotransplantation of porcine chondrocytes into rabbits was unsuccessful for long term cartilage healing [11].

Our study provided a unique contribution to the relatively limited body of work in the literature using this type of co-culture model. Our data is in agreement with previous literature suggesting that mesenchymal stromal cells maybe immunotolerated or immunoprivileged [15, 17,33]. Findings in our human allogeneic experiment demonstrated that no detectable immune activation was prompted; however, an immunosuppression was not observed as in other studies. In cell culture, MSCs have also been able to suppress $\mathrm{T}$ cell proliferation [13], sug- 
gesting MSC, including stem cells, are not only immunotolerated, but may be immunosuppressive [15]. Maccario and colleagues observed a decrease in the formation of $\mathrm{CD}^{+} \mathrm{T}$ cells and natural killer lymphocytes in favor of an expansion of $\mathrm{CD}^{+}$lymphocytes in response to allogeneic MSC [17]. However, in our xenogeneic experiments, the evidence suggested that equine dermal fibroblasts may play a role in the inhibition of an immune response. Our data showed less of an increase in $\mathrm{CD}^{+}$cells after exposure to xenogeneic dermal fibroblasts. Additional evidence has shown that fibroblasts, both autologous or allogeneic cells, resulted in no increase in T-cell proliferation; however, this effect required a modification of CD80 expression on the surface of the cell [12]. Data is accumulating that suggests an immunotolerance to selected allogeneic and xenogeneic isolated cells.

Our data showed variability in human splenocyte initial cell proportions from human to human, demonstrated as wide standard deviations in isolated hSpl numbers and percentages of $\mathrm{CD}^{+}$cells. The variability of splenocyte populations under our conditions are likely explained by differences in age, gender, and hematological parameters of our human spleen donors. Human spleens were acquired from an organ donor center, without exclusion for age, gender, or cause of death. Previous health history of the human spleen donors may have altered the proportion of various cell types in the spleen including mature lymphocytes, T-helper cells, dendritic cells, or natural killer/ cytotoxic T cells. The heterogeneous human population providing hSpl sources probably added variability to our co-culture condition responses, but represent a typical recipient population of humans. Despite the variability inherent in using hSpl from multiple human donors, we were able to demonstrate the natural selection and adaptation of hSpl to the cell culture environment in a shortterm cell culture study, minimal lymphocyte activation by allogeneic and xenogeneic cell exposure and the ability of donor allogeneic and xenogeneic cells to thrive in direct contact with a mixed population of immune cells.

The clinical applicability of our work extends to potential cell-based strategies for the repair of human tissue. Allogeneic or xenogeneic cell sources could serve as a powerful clinical tool to enhance current medical practices that promote healing. Finding a potential cell- or tissue-based treatment for cartilage is dependent upon further understanding of the immunoresponse of cells, including synovial lining cells or chondrocytes. Further characterizing the immunoresponse may enable potential molecular or other cellular modifications to enable specific cells to "tolerate" an immune response or modify the native immune response. It is possible that specific cells could be engineered to modify the acute immune response in vivo, ahead of or in conjunction cellular in- jection or transplantation

\section{Conclusions}

We uniquely investigated the immunoresponse of a mixed population of hSpl to three different mechanisms of allogeneic or xenogeneic challenge: 1) cellular antigens 2) cellular/adenoviral antigens and 3) lipopolysaccharide (LPS). In our in vitro model, neither allogeneic nor xenogeneic cells elicited a lymphocyte/dendritic cell immunoresponse. Our study provided evidence that, in a shortterm culture environment, hSpl undergo a natural selection and adaptation to cell culture conditions. In hSpl cocultures with hSD-MSC, hSD-MSC-GFP, eqDFb, or eqMSC, $\mathrm{CD}^{+}$and $\mathrm{CD}^{+} \mathrm{hSpl}$ increased in proportion and decreased in number with time in culture, but not with exposure to allogeneic or xenogeneic cell antigens. The evidence suggested that allogeneic or xenogeneic may be immunotolerated in the short term and upon first exposure by human recipients. Further studies to characterize the long-term response in vivo and the anemnestic response is warranted

\section{Acknowledgements}

Dr. Jump was supported by a Fellowship at The Ohio State University from the Sports Medicine Center. Dr. Bertone was supported by NIH/NIAMS grant number K08AR4920101. The work was supported by the Trueman Endowment. We would like to acknowledge and thank Dr. Akikazu Ishihara for his assistance in the statistical and data analysis and for proofreading and editing our mansucript. Additionally, we offer a special thanks to Dr. Prosper Boyaka and his laboratory personnel, Dr. Junbae Jee and Haley Steiner, for assistance with technical guidance and methodological expertise in performing flow cytometry.

\section{REFERENCES}

[1] R. E. Newman, D. Yoo, M. A. LeRoux and A. Danilkovitch-Miagkova, "Treatment of Inflammatory Diseases with Mesenchymal Stem Cells," Inflammation \& Allergy_Drug Targets, Vol. 8, No. 2, 2009, pp. 110-123. doi:10.2174/187152809788462635

[2] M. Brittberg, “Autologous Chondrocyte Transplantation,” Clinical Orthopaedics Related Research, Vol. 367, 1999, pp. 147-155. doi:10.1097/00003086-199910001-00016

[3] M. Brittberg, "Autologous Chondrocyte ImplantationTechnique and Long-Term Follow-Up,” Injury, Vol. 39 No. 1, pp. 40-49. doi:10.1016/j.injury.2008.01.040

[4] P. D. Gikas, L. Bayliss, G. Bentley and T. W. Briggs, "An Overview of Autologous Chondrocyte Implantation," The Journal of Bone \& Joint Surgery (British Volume), Vol. 91, 2009, pp. 997-1006. 


\section{doi:10.1302/0301-620X.91B8.21824}

[5] J. D. Harris, R. A. Siston, X. Pan and D. C. Flanigan, "Autologous Chondrocyte Implantation: A Systematic Review," The Journal of Bone \& Joint Surgery, Vol. 92, No. 12, 2010, pp. 2220-2233. doi:10.2106/JBJS.J.00049

[6] D. G. Jones and L. Peterson, "Autologous Chondrocyte Implantation," Instructional Course Lecturers, Vol. 56, 2007, pp. 429- 445.

[7] H. Nakamura, M. Tanaka, K. Masuko-Hongo, et al., "Enhanced Production of MMP-1, MMP-3, MMP-13, and RANTES by Interaction of Chondrocytes with Autologous T Cells," Rheumatology International, Vol. 26, No. 11, 2006, pp. 984-990. doi:10.1007/s00296-006-0116-5

[8] P. A. Revell, V. Mayston, P. Lalor and P. Mapp, "The Synovial Membrane in Osteoarthritis: A Histological Study Including the Characterisation of the Cellular Infiltrate Present in Inflammatory Osteoarthritis Using Monoclonal Antibodies, "Annals of the Rheumatic Diseases, Vol. 47, No. 4, 1988, pp. 300-307. doi:10.1136/ard.47.4.300

[9] L. I. Sakkas, C. Scanzello, N. Johansom, et al., "T Cells and T-Cell Cytokine Transcripts in the Synovial Membrane in Patients with Osteoarthritis, "Clinical Vaccine Immunology, Vol. 5, No. 4, 1998, pp. 430-437.

[10] M. Pei, F. He, B. M. Boyce and V. L. Kish, "Repair of Full-Thickness Femoral Condyle Cartilage Defects Using Allogeneic Synovial Cell-Engineered Tissue Constructs,” Osteoarthritis and Cartilage, Vol. 17, No. 6, 2009, pp. 714-722. doi:10.1016/j.joca.2008.11.017

[11] M. Pei, Z. Yan, M. Shoukry and B. M. Boyce, "Failure of Xenoimplantation Using Porcine Synovium-Derived Stem Cell-Based Cartilage Tissue Constructs for the Repair of Rabbit Osteochondral Defects,"Journal Orthopaedic Research, Vol. 28, No. 8, 2010, pp. 1064-1070.

[12] V. M. Corrigall, E. Solau-Gervais and G. S. Panayi, "Lack of CD80 Expression by Fibroblast-Like Synoviocytes Leading to Anergy in T Lymphocytes," Arthritis \& Rheumatism, Vol. 43, No. 7, 2000, pp. 1606-1615. doi:10.1002/1529-0131(200007)43:7<1606::AID-ANR26 $>3.0 . \mathrm{CO} ; 2-\mathrm{O}$

[13] F. Djouad, V. Fritz, F. Apparailly, et al., "Reversal of the Immunosuppressive Properties of Mesenchymal Stem Cells by Tumor Necrosis Factor Alpha in Collagen-Induced Arthritis,” Arthritis \& Rheumatism, Vol. 52, No. 5, 2005, pp. 1595-1603. doi:10.1002/art.21012

[14] N. Nikolaeva, E. Uss, E. M. van Leeuwen, R. A. van Lier and I. J. ten Berge, "Differentiation of Human Alloreactive CD4+ and CD8+ T Cells in Vitro, " Transplantation, Vol. 78, No. 6, 2004, pp. 815-824. doi:10.1097/01.TP.0000133308.60226.FA

[15] P. Renner, E. Eggenhofer, A. Rosenauer, et al., "Mesenchymal Stem Cells Require a Sufficient, Ongoing Immune Response to Exert Their Immunosuppressive Function,” Transplantation Proceeding, Vol. 41, No. 6, 2009, pp. 2607-2611. doi:10.1016/j.transproceed.2009.06.119

[16] P. Renner, F. C. Popp, E. Eggenhofer, et al., “AntigenSpecific Recognition Is Critical for the Function of Regulatory CD8 ${ }^{+} \mathrm{CD} 28^{-} \mathrm{T}$ Cells," Transplant Immunology, Vol. 22, No. 3-4, 2010, pp. 144-149. doi:10.1016/j.trim.2009.10.002
[17] R. Maccario, M. Podestá, A. Moretta, et al., "Interaction of Human Mesenchymal Stem Cells with Cells Involved in Alloantigen-Specific Immune Response Favors the Differentiation of CD4+ T-Cell Subsets Expressing a Regulatory/Suppressive Phenotype," Haematologica, Vol. 90, No. 4, 2005, pp. 516-525.

[18] L. A. Fortier, A. J. Nixon, J. Williams and C. S. Cable, "Isolation and Chondrocytic Differentiation of Equine Bone Marrow-Derived Mesenchymal Stem Cells," American Journal of Veterinary Research, Vol. 59, No. 9, 1998, pp. 1182-1187.

[19] A. Ishihara, T. A. Zachos, J. S. Bartlett and A. L. Bertone, "Evaluation of Permissiveness and Cytotoxic Effects in Equine Chondrocytes, Synovial Cells, and Stem Cells in Response to Infection with Adenovirus 5 Vectors for Gene Delivery," American Journal of Veterinary Research, Vol. 67, No. 7, 2006, pp. 1145-1155. doi:10.2460/ajvr.67.7.1145

[20] A. Ishihara, L. J. Zekas, A. S. Litsky, S. E. Weisbrode and A. L. Bertone, "Dermal Fibroblast-Mediated BMP2 Therapy to Accelerate Bone Healing in an Equine Osteotomy Model, " Journal of Orthopaedic Research, Vol. 28, No. 3, 2010, pp. 403-411.

[21] T. A. Zachos, K. M. Shields and A. L. Bertone, "GeneMediated Osteogenic Differentiation of Stem Cells by Bone Morphogenetic Proteins-2 or -6, ” Journal of Orthopaedic Research, Vol. 24, No. 6, 2006, pp. 1279-1291. doi:10.1002/jor.20068

[22] A. Ishihara, L. J. Zekas, S. E. Weisbrode and A. L. Bertone, "Comparative Efficacy of Dermal Fibroblast-Mediated and Direct Adenoviral Bone Morphogenetic Protein-2 Gene Therapy for Bone Regeneration in an Equine Rib Model, " Gene Therapy, Vol. 17, 2010, pp. 733-744. doi:10.1038/gt.2010.13

[23] C. H. Jo, H. J. Ahn, H. J. Kim, S. C. Seong and M. C. Lee, "Surface Characterization and Chondrogenic Differentiation of Mesenchymal Stromal Cells Derived from Synovium, ” Cytotherapy, Vol. 9, No. 4, 2007, pp. 316-327. doi:10.1080/14653240701291620

[24] K. B. Van Landuyt, E. A. Jones, D. McGonagle, F. P. Luyten and R. J. Lories, "Flow Cytometric Characterization of Freshly Isolated and Culture Expanded Human Synovial Cell Populations in Patients with Chronic Arthritis," Arthritis Research \& Therapy, Vol. 12, 2010, p. R15. doi:10.1186/ar2916

[25] P. N. Boyaka, A. Tafaro, R. Fischer, et al., "Effective Mucosal Immunity to Anthrax: Neutralizing Antibodies and Th Cell Responses Following Nasal Immunization with Protective Antigen,” Journal of Immunology, Vol. 170, No. 11, 2003, pp. 5636-5643.

[26] T. Fukuiwa, S. Sekine, R. Kobayashi, et al., "A Combination of Flt3 ligand cDNA and CpG ODN as Nasal Adjuvant Elicits NALT Dendritic Cells for Prolonged Mucosal Immunity," Vaccine, Vol. 26, No. 37, 2008, pp. 4849-4859. doi:10.1016/j.vaccine.2008.06.091

[27] H. D. Adkisson, C. Milliman, X. Zhang, et al., "Immune Evasion by Neocartilage-Derived Chondrocytes: Implications for Biologic Repair of Joint Articular Cartilage," Stem Cell Research, Vol. 4, No. 1, 2010, pp. 57-68. doi:10.1016/j.scr.2009.09.004 
[28] C. J. Centeno, D. Busse, J. Kisiday, et al., "Regeneration of Meniscus Cartilage in a Knee Treated with Percutaneously Implanted Autologous Mesenchymal Stem Cells,” Medical Hypotheses, Vol. 71, No. 6, 2008, pp. 900-908. doi:10.1016/j.mehy.2008.06.042

[29] M. Horie, I. Sekiya, T. Muneta, et al., "Intra-Articular Injected Synovial Stem Cells Differentiate into Meniscal Cells Directly and Promote Meniscal Regeneration without Mobilization to Distant Organs in Rat Massive Meniscal Defect," Stem Cells, Vol. 27, No. 4, 2009, pp. 878887. doi:10.1634/stemcells.2008-0616

[30] Q. V. Jichen, G. Che, G. Jiang, et al., "Immune Suppression Produced by Intrathymic Inoculation with Xenogeneic Antigen and Whole-Body $\gamma$-Irradiation in a Pig-toMonkey Heart Transplantation Model," Transplantation Proceeding, Vol. 42, No. 9, 2010, pp. 3759-3762. doi:10.1016/j.transproceed.2010.06.040
[31] Y. Wang, X. Che, M. A. Armstrong and G. Li, "Survival of Bone Marrow-Derived Mesenchymal Stem Cells in a Xenotransplantation Model,” Journal Orthopaedic Research, Vol. 25, No. 7, 2007, pp. 926-932. doi:10.1002/jor.20385

[32] J. L. C. van Susante, P. Buma, L. Schuman, et al., "Resurfacing Potential of Heterologous Chondrocytes Suspended in Fibrin Glue in Large Full-Thickness Defects of Femoral Articular Cartilage: An Experimental Study in the Goat,” Biomaterials, Vol. 20, No. 13, 1999, pp. 11671175. doi:10.1016/S0142-9612(97)00190-7

[33] F. Djouad, C. Bony, T. Häupl, et al., "Transcriptional Profiles Discriminate Bone Marrow-Derived and Synovium-Derived Mesenchymal Stem Cells," Arthritis Research \& Therapy, Vol. 7, 2005, pp. R1304-R1315. doi:10.1186/ar1827 